\title{
Predicting the Diameters of Droplets Produced in Turbulent Liquid-Liquid Dispersion
}

\author{
John Thomas ${ }^{1}$, Brian DeVincentis ${ }^{1}$, Johannes Wutz ${ }^{2}$, and Francesco Ricci ${ }^{3}$ \\ ${ }^{1}$ M-Star Simulations \\ ${ }^{2}$ M-Star Center Europe \\ ${ }^{3}$ Boehringer Ingelheim Corp USA
}

August 7, 2021

\begin{abstract}
The droplet size distribution in liquid-liquid dispersions is a complex convolution of impeller speed, impeller type, fluid properties, and flow conditions. In this work, we present three a priori modeling approaches for predicting the droplet diameter distributions as a function of system operating conditions. In the first approach, called the two-fluid approach, we use highresolution solutions to the Navier-Stokes equations to directly model the flow of each phase and the corresponding droplet breakup/coalescence events. In the second approach, based on an Eulerian-Lagrangian model, we describe the dispersed fluid as individual spheres undergoing ongoing breakup and coalescence events per user-defined interaction kernels. In the third approach, called the Eulerian-Parcel model, we model a sub-set of the droplets in the Eulerian-Lagrangian model to estimate the overall behavior of the entire droplet population. We discuss output from each model within the context of predictions from first principles turbulence theory and measured data.
\end{abstract}

\section{Hosted file}

aichej2021.pdf available at https://authorea.com/users/429537/articles/533214-predicting-thediameters-of-droplets-produced-in-turbulent-liquid-liquid-dispersion 